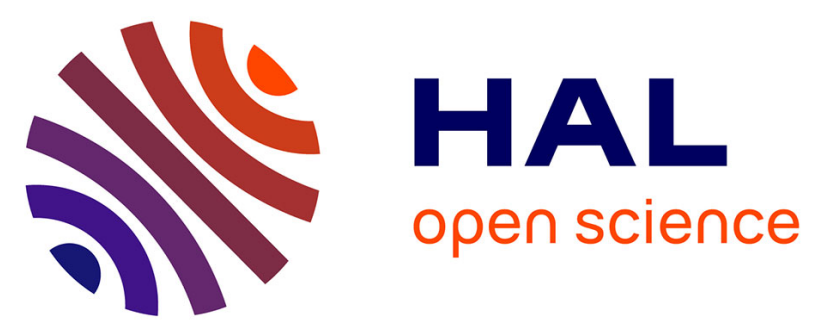

\title{
20-Gbps QPSK signal generation using a silicon dual-drive Mach-Zehnder modulator operating in the O-Band
}

\author{
Laurent Bramerie, Diego Perez-Galacho, Charles Baudot, Mohamed E Chaibi, \\ Sonia Messaoudène, Nathalie Vulliet, Laurent Vivien, Delphine Marris-Morini, \\ Christophe Peucheret
}

\section{To cite this version:}

Laurent Bramerie, Diego Perez-Galacho, Charles Baudot, Mohamed E Chaibi, Sonia Messaoudène, et al.. 20-Gbps QPSK signal generation using a silicon dual-drive Mach-Zehnder modulator operating in the O-Band. 43rd European Conference on Optical Communication (ECOC 2017), Sep 2017, Gothenburg, Sweden. pp.Th.1.C.2, 10.1109/ECOC.2017.8346038 . hal-01609722

\section{HAL Id: hal-01609722 https://hal.science/hal-01609722}

Submitted on 3 Oct 2017

HAL is a multi-disciplinary open access archive for the deposit and dissemination of scientific research documents, whether they are published or not. The documents may come from teaching and research institutions in France or abroad, or from public or private research centers.
L'archive ouverte pluridisciplinaire HAL, est destinée au dépôt et à la diffusion de documents scientifiques de niveau recherche, publiés ou non, émanant des établissements d'enseignement et de recherche français ou étrangers, des laboratoires publics ou privés. 


\title{
20-Gbps QPSK Signal Generation Using a Silicon Dual-Drive Mach-Zehnder Modulator Operating in the O-Band
}

\author{
L. Bramerie(1), D. Pérez Galacho(2), C. Baudot(3), M. Chaibi(1), S. Messaoudène(3), N. Vulliet( ${ }^{(3)}$, \\ L. Vivien ${ }^{(2)}$, D. Marris-Morini(2) and C. Peucheret(1) \\ (1) FOTON Laboratory, CNRS, University of Rennes 1, 22300 Lannion, France \\ laurent.bramerie@enssat.fr \\ (2) Centre de Nanosciences et de Nanotechnologies, CNRS, Univ. Paris-Sud, Université Paris-Saclay, \\ C2N-Orsay, 91405 Orsay Cedex, France \\ (3) STMicroelectronics, 850 rue Jean Monnet, 38920 Crolles, France
}

\begin{abstract}
Gbps QPSK modulation in the O-band is experimentally demonstrated using a simple transmitter structure based on a silicon dual-drive Mach-Zehnder modulator. A power penalty of only $1.5 \mathrm{~dB}$ was obtained with respect to the same transmitter structure in $\mathrm{LiNbO}_{3}$.
\end{abstract}

\section{Introduction}

Silicon optical modulators are of high interest in the context of short-range optical fiber communication systems such as access and data center networks, where their reduced footprint and power consumption as well as their potentially low cost are highly attractive. In such systems, the use of the O-band $(1260 \mathrm{~nm}-$ $1360 \mathrm{~nm}$ ) is an interesting option in order to benefit from the low dispersion of standard single-mode fibers compared to the $\mathrm{C}$-band $(1530 \mathrm{~nm}-1565 \mathrm{~nm})$ and thus be able to increase the bit rate without having to compromise on the distance.

Among different options implementing phaseshifters required for either ring resonator modulators (RRMs), or Mach-Zehnder modulators (MZMs), the use of the free-carrier plasma dispersion effect through carrier depletion in lateral PN junctions results in an interesting trade-off between modulation efficiency, insertion loss and electro-optical bandwidth. However, this effect is somehow weaker in the O-band than in the C-band ${ }^{1}$.

In order to provide higher capacity, it is anticipated that, following the evolution of longhaul optical systems, short reach applications will also make use of advanced optical modulation formats such as $n$-level pulse amplitude modulation (PAM- $n$ ), quadrature phase-shift keying (QPSK) and quadrature amplitude modulation (QAM). There is therefore a strong current interest for the generation of such advanced formats with silicon modulators. For instance, 56-Gbps PAM-4 has been demonstrated in a two-segment MZM², 112-Gbps polarization-multiplexed QPSK has been obtained with an in-phase and quadrature (IQ) modulator $^{3}$ while 80 -Gbps 16 -QAM has been demonstrated by using a two-branch modulator with two MZMs in each branch ${ }^{4}$. Those modulator structures are however quite complex.

The use of a single dual-drive Mach-Zehnder modulator (DD-MZM) to generate QPSK modulation has been proposed ${ }^{5}$ and demonstrated using $\mathrm{LiNbO}_{3}$ modulators ${ }^{6,7}$. The scheme is well suited for lower-efficiency phaseshifters, since it only requires a peak-to-peak voltage of $V_{\pi}$ (half-wave voltage) to be applied to each phase-shifter, instead of $2 V_{\pi}$ in an IQ modulator. It also benefits from a simpler modulator structure, although at the expense of stronger requirements on the quality and control of amplitude of the driving signals.

In this work, we demonstrate for the first time 20-Gbps QPSK signal generation using a silicon DD-MZM operating in the O-band. We furthermore compare its performance with the same implementation making use of a commercial 28-GHz $\mathrm{LiNbO}_{3}$ DD-MZM and discuss the relative performance of the scheme with respect to a conventional IQ modulatorbased transmitter.

\section{Modulator design and principle of operation}

(a)

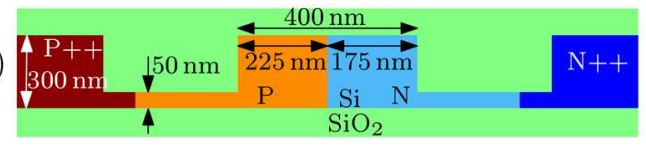

(b) Input splitter

Fig. 1: (a) Cross section of the PN junction-based phase-shifter and (b) schematic representation of the dual-drive Mach-Zehnder modulator.

The modulator was fabricated using the $300-\mathrm{mm}$ technological platform of STMicroelectronics. The cross-section of the designed phase-shifter is represented in Fig. 1(a). It consists in a PN junction implemented in a rib waveguide and was 
designed following a simplified modeling approach $^{8}$. The targeted doping concentrations of the junction were $p=5 \times 10^{17} \mathrm{~cm}^{-3}$ and $n=1.4 \times 10^{18} \mathrm{~cm}^{-3}$ for the holes and the electrons, respectively.

The phase-shifter was integrated in both arms of a Mach-Zehnder interferometer in order to form a DD-MZM, as schematically depicted in Fig. 1(b), in which the $\Delta n_{i}$ boxes represent the phase-shifting elements and the $\Phi$ box symbolizes a constant phase-shift intended to set the operation point of the interferometer.

To generate a QPSK signal with a single DD$M Z M$, two independent binary modulating signals are applied to the phase-shifters in order to achieve a peak-to-peak phase modulation of $\pi$ in each arm. The constant phase-shift is then adjusted to bias the interferometer at a quadrature point $(\Phi=\pi / 2)$. In contrast with conventional QPSK transmitters using an IQ modulator, the DD-MZM is not biased to null transmission.

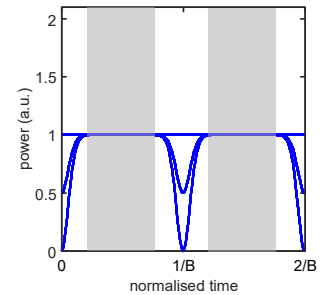

(a)

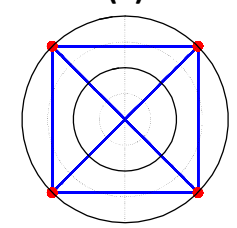

Fig. 2: Eye (top) and constellation (bottom) diagrams for QPSK signals generated with (a) an IQ modulator and (b) a DD-MZM.

Theoretical eye and constellation diagrams obtained for QPSK modulation performed with IQ and DD-MZM modulators are represented in Fig. 2(a) and (b), respectively, where the differences between signals generated using the two schemes are highlighted. First, the DD-MZM results in more intensity ripples linked to symbol transitions compared to the IQ modulator. Second, the shaded areas in the eye diagrams of Fig. 2 represent areas where the intensity is constant, hence that are suitable for sampling and phase recovery. The DD-MZM presents reduced temporal opening than the IQ modulator and is thus more sensitive to symbol transitions. These apparent drawbacks are however compensated by a much simpler implementation and the need for reduced driving voltages.

\section{Experimental setup}

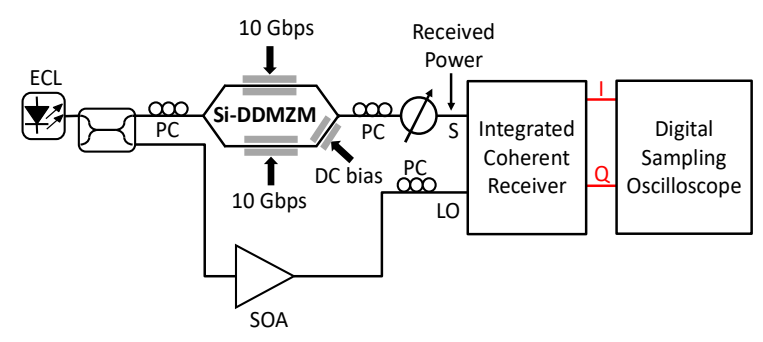

Fig. 3: Experimental setup.

The experimental setup is represented in Fig. 3 . TE-polarized light from an O-band external cavity laser (ECL) was injected in the fabricated chip using a grating coupler. Two $2^{15}-1$ long decorrelated pseudo random binary sequences (PRBSs) at $10 \mathrm{Gbps}$ were used as modulating signals. The modulating signals were amplified to $7 \mathrm{Vpp}$ and a DC bias of $4 \mathrm{~V}$ was added to ensure that the PN junctions were operated in depletion. The modulated optical signal was then input to an integrated coherent receiver through a variable optical attenuator in order to adjust the received power. For the purpose of this first demonstration, the same laser was used at the transmitter and as local oscillator (LO) at the receiver, thus implementing homodyne detection. A semiconductor optical amplifier (SOA) was used to amplify the LO signal.

The I/Q signals at the output of the receiver front-end were fed to a $40 \mathrm{GSps}$ digital sampling oscilloscope (DSO) with 16-GHz electrical bandwidth. Blocks of $10^{5}$ symbols were acquired and then processed offline. For digital processing, an adaptive finite impulse response (FIR) filter with direct-decision least mean square (DD-LMS) tracking was used. The processed samples were used to represent the signal constellations and to compute the bit-error ratio (BER). Each BER measurement is the result of averaging over five $10^{5}$ symbol-long data blocks.

In order to benchmark its performance, the characterizations were repeated after replacing the silicon DD-MZM with a $28-\mathrm{GHz} \mathrm{LiNbO}_{3} \mathrm{DD}-$ MZM and a 25-GHz $\mathrm{LiNbO}_{3} \mathrm{IQ}$ modulator.

\section{Results and discussion}

The silicon DD-MZM was first characterized without modulating signals. The measured fiberto-fiber attenuation was $17 \mathrm{~dB}$, including the losses of the grating couplers, on-chip passive losses and modulator insertion loss (IL). Using a straight waveguide on the chip as normalization, the IL of the modulator was estimated to be $2.5 \mathrm{~dB}$. The half-wave voltage of each phaseshifter was measured to be between $6 \mathrm{~V}$ and $7 \mathrm{~V}$, which, taking into account the 2-mm active length, leads to modulation efficiencies between 
$1.2 \mathrm{~V} \cdot \mathrm{cm}$ and $1.4 \mathrm{~V} \cdot \mathrm{cm}$.
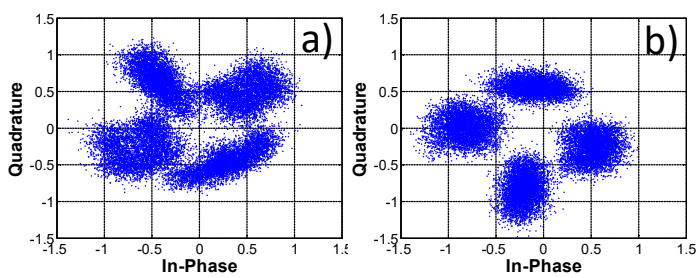

Fig. 4: Unprocessed constellation diagrams for (a) silicon and (b) $\mathrm{LiNbO}_{3}$ DD-MZM ( $\left.P_{\mathrm{rx}}=-22 \mathrm{dBm}\right)$

The raw constellations diagrams of $20-\mathrm{Gbps}$ QPSK signals generated with silicon and $\mathrm{LiNbO}_{3}$ DD-MZMs are shown in Fig. 4(a) and (b), respectively. They are obtained for the same receiver input power of $-22 \mathrm{dBm}$ from 2000 samples after resampling, decimation to the baud rate, but without digital equalization. Four sample clusters corresponding to the four phase states can be clearly identified. The particular shape of this QPSK constellation is linked to optical intensity ripples between consecutive symbols inherent to the DD-MZM generation method. Bandwidth limitations result in intensity fluctuations linked to particular trajectories of symbol transitions, as expected from Fig. 2(b).
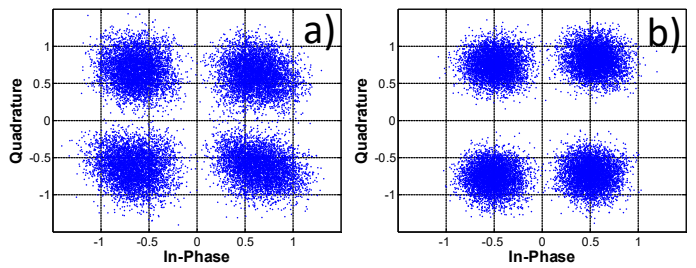

Fig. 5: Constellation diagrams after DSP for (a) silicon and (b) $\mathrm{LiNbO}_{3} \mathrm{DD}-\mathrm{MZM}\left(P_{\mathrm{rx}}=-22 \mathrm{dBm}\right)$.

After complete digital signal processing (DSP), classical QPSK constellations can be retrieved, as shown in Fig. 5. Error vector magnitudes (EVMs) of $33 \%$ and $27 \%$ were measured at $-22 \mathrm{dBm}$ receiver input power for silicon and $\mathrm{LiNbO}_{3}$ modulators, respectively. This small EVM degradation observed with the silicon modulator is related to intensity ripples induced by its lower bandwidth $(15 \mathrm{GHz})$ compared to the $\mathrm{LiNbO}_{3}$ modulator.

The BER performance is reported as a function of the receiver input power in Fig. 6. All the curves have been obtained with a local oscillator power of $+14 \mathrm{dBm}$. The BER performance for the $\mathrm{LiNbO}_{3} \mathrm{DD}-\mathrm{MZM}$ presents $5 \mathrm{~dB}$ penalty at a BER of $10^{-3}$ with respect to the conventional IQ modulator, which is due to the presence of intensity ripples that are inherent to this modulation scheme, as previously explained. The silicon DD-MZM presents a remarkably low 1.5-dB extra penalty compared to its $\mathrm{LiNbO}_{3}$ counterpart, which is linked to its reduced electrooptical bandwidth of $15 \mathrm{GHz}$.

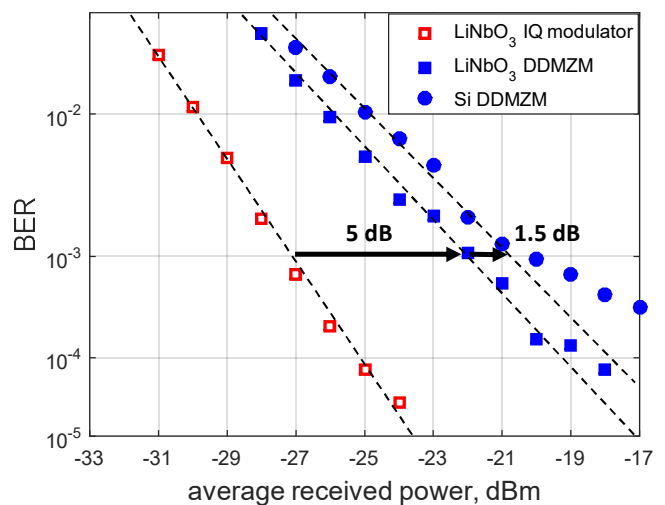

Fig. 6: BER versus received power

\section{Conclusions}

We have reported the first use of a silicon dualdrive Mach-Zehnder modulator to generate a 20-Gbps QPSK signal in the O-band. The feasibility of this approach is demonstrated for the first time in silicon with only $1.5-\mathrm{dB}$ penalty in comparison to a $\mathrm{LiNbO}_{3}$ dual-drive modulator. The penalty compared to an IQ modulator could be further decreased thanks to pre-emphasis of the electrical drive voltages ${ }^{6}$ or with an optical pulse carver ${ }^{7}$.

\section{Acknowledgements}

This work was supported by the European project Plat4m (FP7-2012-318178) and the European project Cosmicc (H2020-ICT-27-2015-688516). Support of French Industry Ministry/DGE through the Nano2017 program is also acknowledged.

\section{References}

[1] G. T. Reed et al. "Silicon optical Modulators," Nat. Photonics, Vol. 4, no. 8, p. 518 (2010).

[2] C. Xiong et al., "Monolithic $56 \mathrm{~Gb} / \mathrm{s}$ silicon photonic pulseamplitude modulation transmitter," Optica, Vol. 3, no. 10, p. 1060 (2016)

[3] P. Dong et al. "112-Gb/s monolithic PDM-QPSK modulator in silicon" Opt. Express, vol. 20, no. 26, p. B624 (2012).

[4] J. Ding et al., "Silicon 16-QAM optical modulator driven by four binary electrical signals," Opt. Letters, Vol. 42, no. 8, p. 1636 (2017)

[5] K.-P. Ho and H.-W. Cuei, "Generation of arbitrary quadrature signals using one dual-drive modulator" J. Lightw. Technol, Vol. 23, no. 2, p. 764 (2005).

[6] D. J. Krause et al., "Demonstration of 20-Gb/s DQPSK with a single dual-drive Mach-Zehnder modulator". IEEE Photon. Technol. Lett., Vol. 20, no. 16, p. 1363 (2008).

[7] W.-R. Peng et al., "Evaluation of the inherent ripple effects on the performance of an optical DQPSK signal generated by using only one MZM“, Proc. Photonics in Switching, Heraklion (2006)

[8] D. Pérez-Galacho et al., "Simplified modeling and optimization of silicon modulators based on free-carrier plasma dispersion effect," Opt. Express, Vol. 24, no. 23, p. 26332 (2016). 